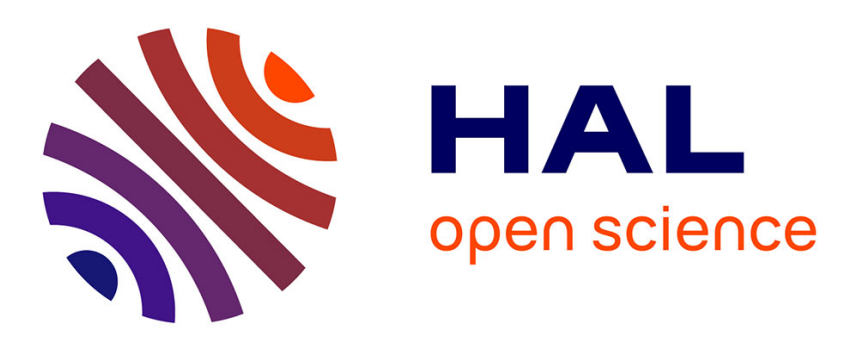

\title{
Performance evaluation of Multi-User MIMO Underwater Acoustic Communications
}

\author{
Antony Pottier, Pierre-Jean Bouvet, Philippe Forjonel
}

\section{To cite this version:}

Antony Pottier, Pierre-Jean Bouvet, Philippe Forjonel. Performance evaluation of Multi-User MIMO Underwater Acoustic Communications. 2021 fifth Underwater Communications and Networking Conference (UComms), Aug 2021, Lerici, Italy. hal-03376552

\section{HAL Id: hal-03376552 https://hal.science/hal-03376552}

Submitted on 13 Oct 2021

HAL is a multi-disciplinary open access archive for the deposit and dissemination of scientific research documents, whether they are published or not. The documents may come from teaching and research institutions in France or abroad, or from public or private research centers.
L'archive ouverte pluridisciplinaire HAL, est destinée au dépôt et à la diffusion de documents scientifiques de niveau recherche, publiés ou non, émanant des établissements d'enseignement et de recherche français ou étrangers, des laboratoires publics ou privés. 


\title{
Performance evaluation of Multi-User MIMO Underwater Acoustic Communications
}

\author{
Antony Pottier, Pierre-Jean Bouvet, Philippe Forjonel \\ Institut Suprieur d'Electronique et du Numrique (ISEN), Yncrea Ouest, LabISEN \\ 20 Rue Cuirass Bretagne, 29200 Brest, France \\ Email: \{antony.pottier,pierre-jean.bouvet,philippe.forjonel\} @isen-ouest.yncrea.fr
}

\begin{abstract}
This paper presents a study of Multiuser Multiple Input Multiple Output (MU-MIMO) as a multi-access method for underwater acoustic communications. Traditional multi-access schemes such as Time Division Multiple Access (TDMA) suffer from limited data rates due to an inefficient sharing of communication resources. In a MU-MIMO systems, each user can be viewed by the receiver as an input of a conventional MIMO channel. The receiver can then exploit the spatial diversity to decode the input streams of each users. Achievable rates of MU-MIMO with Successive Interference Cancellation (SIC) are studied in a first place, and compared with single user and TDMA theoretical performance. The theoretical rates are computed on channels sounded at-sea for a two-user configuration so as to evaluate what gains can be expected from the MU-MIMO approach. Finally, comparisons with the effective rates obtained from simulations of full modulation and demodulation stages using measured channels and the underWater AcousTic channEl Replay benchMARK (WATERMARK) are provided.
\end{abstract}

Index Terms-Underwater acoustic communications, achievable rates, MIMO, multiple access, channel replay, successive interference cancellation.

\section{INTRODUCTION}

Underwater Acoustic (UWA) channel is one of the most challenging communication channel due to its doubly-selective fading nature represented by extremely long delay spread and fast time variation [1]. On the other-side, data rates required for UWA communications applications are continuously growing with the introduction of high-quality images and real-time video. Moreover, deployment of sensor networks, seafloor observatories or autonomous fleets of cooperating Autonomous Underwater Vehicle (AUV) require the UWA channel medium to be shared by multiple users [2]. Traditional multi-access technique for UWA communication is TDMA where a specific time slot followed by a guard interval is assigned to a given user in order to avoid multi-user interference. One major drawback of TDMA lies in date rate limitation due to singleuser time slot and guard interval [3].

Recently MU-MIMO approach has been investigated as multiple access technique for UWA communication [4]-[6]. In an uplink MU-MIMO scheme, each user transmit data simultaneously within the same frequency, so it can be viewed as a distributed MIMO system where each user acts as an

This work has been funded by Direction Générale de l'Armement, under the RapidDGA program. Data from channels soundings experiment have been provided by RTSYS - 25 Rue Michel Marion, 56850 Caudan, France. input stream of the channel. At the receive side, by combining multiple channel outputs, the spatial signature uniqueness of each user can be exploited to decode each user stream separately. With respect to TDMA protocol, the MU-MIMO technique enables spatial multiplexing of user data at the prize of multi-user interference terms, that need to be estimated and cancelled at the receiver side. The objective of our paper is to estimate the gain of the MU-MIMO approach over a traditional TDMA approach for a realistic multiuser UWA communication channel by using achievable rate metric, and to compare it against effective data rate obtained with a full modulation and demodulation system. Realistic simulations of UWA transmissions are performed by using the WATERMARK [7] fed by channel soundings performed in Bay of Brest, France.

The paper is organized as follows: Section II provides the computation of theoretical achievable rates for MU-MIMO channels, then Section III details transmitter and receiver structures. Section IV describes the channel sounding and experimental results, before conclusions in Section V.

Notations: Upper case bold letters $\boldsymbol{M}$ denotes matrices, while lowercase bold letters $\boldsymbol{x}$ are used for vectors. The Hermitian transpose of $\boldsymbol{A}$ is denoted by $\boldsymbol{A}^{H}$, and $\boldsymbol{I}_{L}$ is the identity matrix of size $L$. Sets of integers of the form $\{1, \cdots, N\}$ are written $\llbracket 1, N \rrbracket . \mathbb{E}$ stands for expectations. $\mathbb{E}_{t}[X(t, \tau)]$ is expectation according to the realizations of the multivariate random process $X(t, \tau)$ along the dimension indexed by $t$. The cardinal of a set $\mathcal{S}$ is denoted by $\operatorname{Card}(\mathcal{S})$.

\section{ACHIEVABle RATES FOR MU-MIMO UWA COMMUNICATIONS}

We consider $I$ transmitters communicating with a single receiver equipped with $N_{r x}$ hydrophones. The transmitters have a single transducer, they are not co-located, and they transmit their data simultaneously to the receiver using the same bandwidth. In a practical set-up, transmitters could be Autonomous Underwater Vehicles (AUVs) communicating with a central node or buoy for example. Let $\boldsymbol{x}_{i}=\left(x_{i}[0], \cdots, x_{i}[N-1]\right)^{T}$ a frame of $N$ i.i.d complex and zero mean symbols sent by user $i \in \llbracket 1, I \rrbracket$. For any $j \neq i$, the symbols $\boldsymbol{x}_{j}$ sent by any other user $j$ are independent of $\boldsymbol{x}_{i}$. For every user $i \in \llbracket 1, I \rrbracket$, the following power constraint is applied :

$$
\mathbb{E}\left[\boldsymbol{x}_{i} \boldsymbol{x}_{i}^{H}\right]=P \boldsymbol{I}_{N},
$$

with the same transmit power $P$ for every users. 
The focus is first put on the flat fading case for the ease of presentation. The receiver's observation at time $t$ can be expressed by

$$
\boldsymbol{y}[t]=\sum_{i=1}^{I} \boldsymbol{h}_{i}[t] x_{i}[t]+\boldsymbol{w}[t]
$$

where $\boldsymbol{h}_{i}[t] \in \mathbb{C}^{N_{r x}}$ is the Single Input Multiple Output (SIMO) channel from user $i \in \llbracket 1, I \rrbracket$, and $\boldsymbol{w}[t] \sim$ $\mathcal{C N}\left(\mathbf{0}, \sigma^{2} \boldsymbol{I}_{N_{r x}},\right)$ is a white, stationary, Gaussian noise. This model falls in the class of multiple access channels (MAC) in information theory [8], and will serve as the basis for studying the theoretical achievable rates. This is also an abstraction to what we call here MU-MIMO in the sense that the different users act as different channel inputs from the point of view of the receiver. Note that the assumption of a single transmit antenna per user is not restrictive and is made in order to fit with the experiments we conducted.

The channels are random and their coherence times are much smaller than the frame duration. When the channel realizations are known by the receiver, and assuming SIC decoding, the achievable rate is given by [9] :

$$
R_{i}=\mathbb{E}\left[R_{i}^{\Pi}\right]=\sum_{\Pi \in \mathfrak{I}} p_{\Pi} R_{i}^{\Pi}
$$

where

$$
\begin{aligned}
R_{i}^{\Pi} & =\mathbb{E}_{t}\left[\log _{2}\left|\boldsymbol{I}_{N_{r}}+\rho \sum_{j \in \mathfrak{I}_{i}^{\Pi} \cup\{i\}} \boldsymbol{h}_{j}[t] \boldsymbol{h}_{j}[t]^{H}\right|\right] \\
& -\mathbb{E}_{t}\left[\log _{2}\left|\boldsymbol{I}_{N_{r}}+\rho \sum_{j \in \mathfrak{I}_{i}^{\Pi}} \boldsymbol{h}_{j}[t] \boldsymbol{h}_{j}[t]^{H}\right|\right] .
\end{aligned}
$$

and where $\Pi$ is a random variable defining the decoding order and distributed by the probability $p_{\Pi}$ and $\rho=P / \sigma^{2}$ is the Signal to Noise Ratio (SNR). In equation (4), expectations are taken according to the realizations of $\boldsymbol{h}_{j}[t]$ along time $t$, $j \in \llbracket 1, I \rrbracket$. Thus, the channels are assumed stationary for the duration of the frame. We denote by $\mathfrak{I}$ the set of decoding orders given by all possible permutations of $\llbracket 1, I \rrbracket$, which have equal probabilities $p_{\Pi}=\operatorname{Card}(\mathfrak{I})^{-1}$. The set $\mathfrak{I}_{i}^{\Pi}$ is the set of successors of the user $i$ in the decoding order $\Pi$. The idea expressed by (3) and (4) is that the rate of a user $i$ is penalized by the information conveyed by the contributions to the observation $\boldsymbol{y}[t]$ of the users that have not been yet decoded when $i$ is being decoded.

Extending the expressions of achievable rates presented above to frequency selective fading, the channels can be expressed through their time-varying frequency responses: for each user $j \in \llbracket 1, I \rrbracket$ we define a channel vector realization, at time $t$ and for the frequency bin $f$, by $\boldsymbol{h}_{j}[t, f] \in \mathbb{C}^{N_{r x}}$. Assuming that the transmitters spread equally their power across a bandwidth $B$ divided in $N_{\nu}$ frequency bins, the achievable rate of a user $i$ can be expressed by equation (3) with $R_{i}^{\Pi}$ now given by

$$
\begin{aligned}
R_{i}^{\Pi} & =\frac{1}{N_{\nu}} \sum_{f=1}^{N_{\nu}} \mathbb{E}_{t}\left[\log _{2}\left|\boldsymbol{I}_{N_{r}}+\rho \sum_{j \in \mathfrak{I}_{i}^{\Pi} \cup\{i\}} \boldsymbol{h}_{j}[t, f] \boldsymbol{h}_{j}[t, f]^{H}\right|\right] \\
& -\mathbb{E}_{t}\left[\log _{2}\left|\boldsymbol{I}_{N_{r}}+\rho \sum_{j \in \mathfrak{I}_{i}^{\Pi}} \boldsymbol{h}_{j}[t, f] \boldsymbol{h}_{j}[t, f]^{H}\right|\right],
\end{aligned}
$$

and with the same notation for the decoding order.

This last expression will be used for theoretical evaluations of the achievable rates of the MU-MIMO scheme against TDMA and single user schemes, and provide insights regarding the performance gap obtained in practice between these three schemes.

\section{SYSTEM DESCRIPTION}

In this section, we briefly describe the transmitter and receiver structures implemented for evaluation of effective rates in Section IV. The transmitter and receiver processing are the same as those described in [10] but extended to the MU-MIMO case. The same structure is also used for TDMA and single user operations, however the users frames are separated in time slots in TDMA transmissions.

Bit-Interleaved Coded Modulation (BICM) [11] is employed and users transmit bursts of i.i.d. QPSK symbols modulated around a center frequency $f_{0}$. For each user, the frame is constituted of $N_{p}$ pilot symbols from a Pseudo-noise (PN) sequence, followed by $N_{d}$ data symbols and a guard interval of duration $N_{g} \times T$, where $T=1 / R$ is the symbol duration. Prior to modulation, data bits are encoded by a convolutional encoder of rate $R_{c}=1 / 2$ and constraint length 7 , and interleaved by pseudo-random permutations. The encoder polynomial is $[133,171]_{o}$ in octal notation. Symbols are pulse-shaped by a Square Root Raised Cosine (SRRC) cosine filter $g(t)$ with roll-off $\alpha=0.1$. For a given user $i \in \llbracket 1, I \rrbracket$, the transmitted signal is expressed by

$$
s_{i}(t)=\operatorname{Re}\left\{\sqrt{P} \sum_{n} x_{i}[n] g(t-n T) \mathrm{e}^{j 2 \pi f_{0} t}\right\} .
$$

with $P$ the transmission power, supposed equal for each user.

The pilot symbols are used for synchronization as well as for channel estimation using the Least Squares (LS) method. After baseband conversion and matched-filtering of the received signal, a Constant False Alarm Rate (CFAR) detector [12] is used to localise the frame. Then, timing synchronization is performed thanks to a cross-correlation with the pilot signals. Doppler effects due to motion and clock frequency offsets are assumed to be compensated. After synchronization, the channel output sampled at the symbol time is fed to a MIMO iterative decoder based on the turbo-equalization principle [13] : a MIMO demapper and a soft-in soft-out decoder consist in the main two stages of the iterative decoding process. The MIMO demapper includes an Interference Canceler Linear Equalizer (IC-LE) fed by the channel output, the LS channel estimates and the log-likelihood ratios of symbols decoded from previous iterations. In the MU-MIMO scheme where 
each user is an input stream of the MIMO channel, the receiver described here behaves like a successive interference canceller. The interested reader is referred to [10] for a more complete description of the receiver algorithms.

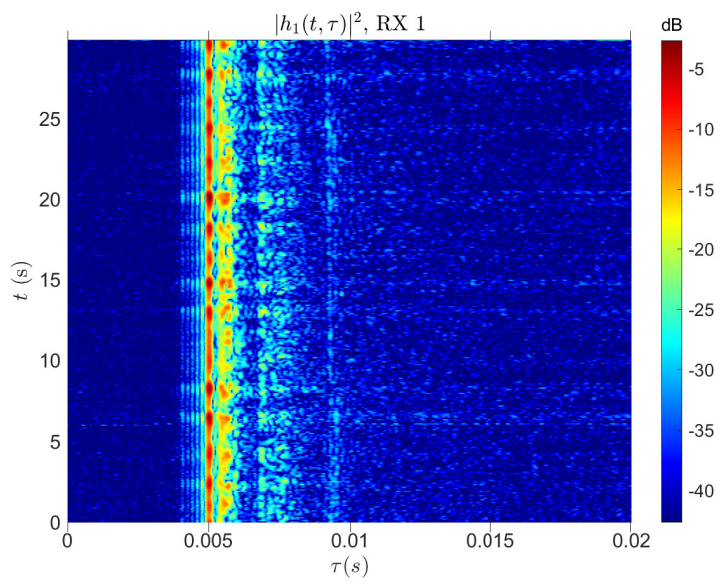

Fig. 1. Time varying channel impulse responses sounded at the first hydrophone of user 1 .

\section{EXPERIMENT DESCRIPTION AND RESULTS}

The MU-MIMO scheme is evaluated for two users $(I=2)$ through channel replay based on channel impulse responses sounded at-sea. Comparisons with TDMA and single user are made with the same channels.

\section{A. Channel soundings}

The channels used for performance evaluation were sounded in the Brest Bay, near Lanvéoc, France, in February 2019. Measurements of the Channel Impulse Response (CIR) were obtained by successive matched filtering to a known probe signal transmitted repeatedly. Probes have been built on the basis of maximum length sequences of 511 BPSK symbols, modulated at a 6 kbauds speed and shaped by a SRRC filter whose roll-off factor is $\alpha=0.1$. Such a sequence covers a channel delay spread of at most $85 \mathrm{~ms}$. The probe is obtained by stacking several pulse-shaped sequences so as to cover an observation time of $T_{o b s}=30 \mathrm{~s}$. Thus, channel estimations are updated every $85 \mathrm{~ms}$, covering Doppler spreads up to $5.88 \mathrm{~Hz}$. Measurements are made in a $6.6 \mathrm{kHz}$ bandwidth centered on a $27 \mathrm{kHz}$ carrier frequency. During the experiment, a transmitter equipped with one transducer immersed at a $2.5 \mathrm{~m}$ depth transmitted the probe to an array of $N_{r x}=5$ hydrophones immersed at $3 \mathrm{~m}$ with a spacing of $1 \mathrm{~m}$, located at approximately 300 meters from the transmitter location. Constant and time-varying Doppler shifts are mitigated at the receiver using the procedure described in [14], [15]. Two soundings were performed at few tens of meters apart, producing two measured SIMO CIR, one for each user of the experiment. Delay and doppler spreads were in the range $9-16$ ms and $0.7-1.15 \mathrm{~Hz}$ respectively, depending on the user and hydrophone. Channel frequency responses averaged over time are depicted in Figure 2 and the time-varying impulse response sounded at the first hydrophone of user 1 in Figure 1.
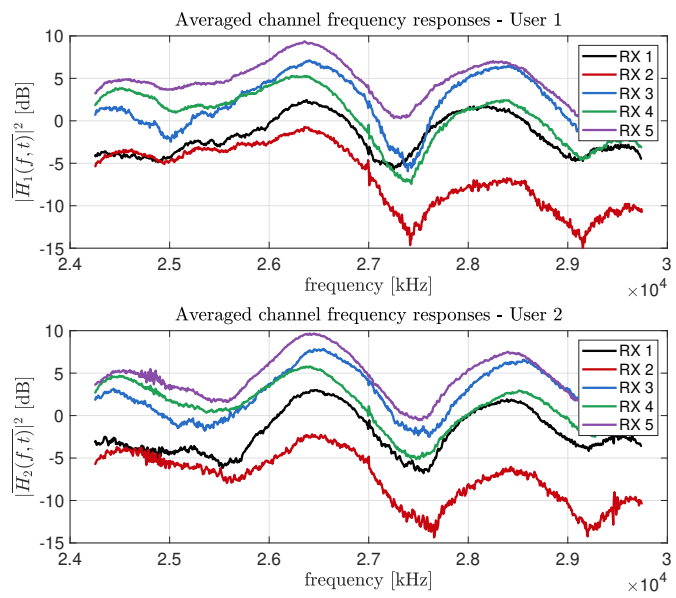

Fig. 2. Average frequency responses of sounded channels (based on 714 realizations of $85 \mathrm{~ms}$ duration).

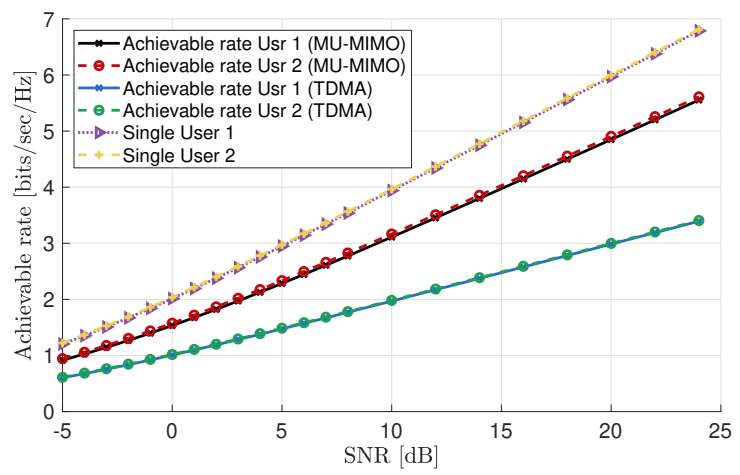

Fig. 3. Achievable rates bounds of MU-MIMO transmissions computed on measured channels, and comparison with TDMA and single user transmissions.

\section{B. Achievable rates on measured channels}

\begin{tabular}{|c|c|c|}
\hline Parameter & Symbol & Value \\
\hline Carrier frequency & $f_{0}$ & $27 \mathrm{kHz}$ \\
Modulation speed & $R$ & $5 \mathrm{kbauds}$ \\
Bandwidth & $B$ & $5.5 \mathrm{kHz}$ \\
Nb data symbols & $N_{d}$ & 1024 \\
Nb pilot symbols (MU-MIMO) & $N_{p}$ & 481 \\
Nb pilot symbols (Single/TDMA) & $N_{p}^{\mathrm{SU}}$ & 241 \\
Nb guard symbols & $N_{g}$ & 250 \\
Nb RX antennas & $N_{r x}$ & 5 \\
Code rate & $R_{c}$ & $1 / 2$ \\
Nb symbols/frame (MU-MIMO) & $N$ & 1755 \\
Nb symbols/frame (Single/TDMA) & $N^{\mathrm{SU}}$ & 1515 \\
Nb of frames/user & $N_{f}$ & 83 \\
\hline
\end{tabular}

TABLE I

TRANSMISSION PARAMETERS

The transmission parameters used for simulations are summed up in Table I. Note that the number of pilots is different in MU-MIMO and in single user or TDMA configuration since we should have $N_{p}>2 I \times \frac{\sigma_{\tau}}{T}-1$ [16] where $\sigma_{\tau}$ is the channel delay spread. The number of symbols per frame in the calculation of rates changes accordingly.

Achievable rates computed on the basis of the two mea- 
sured CIR and for different transmission schemes are drawn in Figure 3. Theoretical performance are shown as spectral efficiencies expressed in bits/s/Hz. Time-varying channel frequency responses of both users were computed through discrete Fourier transformation of the measured CIR along the delay dimension on $N_{\nu}=2048$ points, for each realization and each hydrophone. This produces the channels SIMO vectors $\boldsymbol{h}_{j}[t, f]$ of equation (5), and their averages over time $t$ produce the responses plotted in Figure 2. Based on these channels vectors, the achievable rate $R_{i}$ for $i=1,2$ is computed by averaging over 100 realizations of the random decoding order $\Pi$, and the process is repeated for several values of the noise power $\sigma^{2}$. The channels are normalized according to the hydrophone carrying the highest energy so that the SNR parameter $\rho=1 / \sigma^{2}$ is the effective SNR for this hydrophone, assuming a unit transmission power $P=1$. Spectral efficiencies, as drawned in Figure 3, are computed for each user $i=1,2$ as

$$
\eta_{i}^{\mathrm{MU}-\mathrm{MIMO}}=\frac{N_{d}}{N} \times \frac{2 R_{c} R_{i}}{B T} .
$$

where $R_{i}$ is evaluated through equations (3) and (5), and $R_{c}$, $N_{d}$ and $N$ are given in table I. Spectral efficiencies for TDMA transmission are given by

$$
\eta_{i}^{\mathrm{TDMA}}=\frac{N_{d}}{I \times N^{\mathrm{SU}}} \times \frac{2 R_{c} R_{i}^{\mathrm{SU}}}{B T} .
$$

where

$$
R_{i}^{\mathrm{SU}}=\frac{1}{N_{\nu}} \sum_{f=1}^{N_{\nu}} \mathbb{E}_{t}\left[\log _{2}\left|\boldsymbol{I}_{N_{r}}+\rho \boldsymbol{h}_{i}[t, f] \boldsymbol{h}_{i}[t, f]^{H}\right|\right]
$$

is the achievable rate for the single user $i$, i.e. without interference from $j \neq i$, which is also used to compute the corresponding spectral efficiency in bits/s/Hz :

$$
\eta_{i}^{\mathrm{SU}}=\frac{N_{d}}{N^{\mathrm{SU}}} \times \frac{2 R_{c} R_{i}^{\mathrm{SU}}}{B T} .
$$

The single user bound is obviously twice the TDMA theoretical performance in our two users experiment, since 2 time slots are needed. The MU-MIMO scheme can theoretically perform closer to the single user bound in the channels we measured. At SNRs between 10 and $25 \mathrm{~dB}$, MU-MIMO can be expected to achieve rates equal to $\approx 80 \%$ those of the single user.

\section{Effective data rates on replay channels}

We now evaluate the effective data rates for MU-MIMOSIC, TDMA and single transmissions using the transceiver structure of Section III through measured channels thanks to the WATERMARK channel replay software [7]. Each user is assigned a measured SIMO channel and sends $N_{\text {frames }}=83$ frames. The signals duration cover the channel sounding of 30 s. For each frame and several SNR values, the Frame Error Rate (FER) per user is evaluated. The effective rate for the user $i=1,2$ is then expressed in bits/sec/Hz by :

$$
r_{i}=\frac{N_{d}}{N} \times \frac{1-F E R_{i}}{B T} .
$$

The same formula can be applied for MU-MIMO and single user and is divided by a factor 2 in TDMA with two user

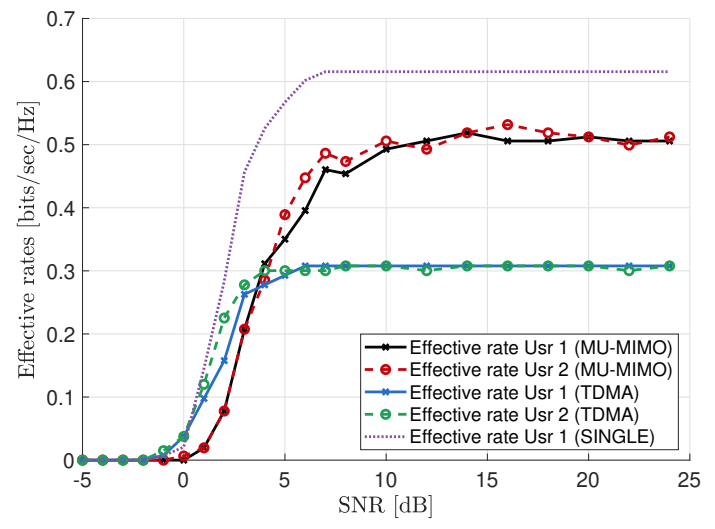

Fig. 4. Effective rates of MU-MIMO transmission computed over WATERMARK channel fed by measured channels, and comparison with TDMA and single-user transmission.

compared to single user, as the frame duration becomes $2 \times$ $N^{\mathrm{SU}} \times T$.

Effective rate are given in Figure 4. In high SNR regimes, MU-MIMO performs closer to the single user effective rate than TDMA. At a $20 \mathrm{~dB}$ SNR, MU-MIMO effective rate $(0.51$ $\mathrm{bits} / \mathrm{s} / \mathrm{Hz})$ is $\approx 83 \%$ the rate of single user $(0.61 \mathrm{bits} / \mathrm{s} / \mathrm{Hz})$, which is the gain expected from the study of the achievable rates. However, because of multi-user interference, the SNR threshold for which MU-MIMO produce non-zero rates is slightly higher than in single user transmissions and TDMA. TDMA offers lower effective rates but a better robustness at low SNR due to the absence of interference.

\section{CONCLUSION}

This paper has presented a study of the MU-MIMO scheme for use in underwater acoustic communications. Instead of separating the different users among orthogonal codes, time or frequency slots, MU-MIMO systems suggest that multiuser transmissions can be viewed as a particular case of conventional MIMO transmissions. This has the potential benefit to bring better spectral efficiencies since the communication resources can be used simultaneously by several users, leaving to the receiver the role of mitigating multi-user interference thanks to SIC decoding. Our conclusions are supported by a study of theoretical and effective rates for MU-MIMO, TDMA and single user transmissions, on the basis of channel sounded at-sea. Theoretic achievable rates show that MU-MIMO with SIC decoding could perform close to the single user bound, compared to TDMA. This performance gap is evaluated experimentally by simulation of the three schemes through channel replay, based on the same transceiver structure employing BICM combined with an iterative receiver. At a sufficiently high SNR $(>10 \mathrm{~dB})$, our results show that MU-MIMO performs at $\approx 80 \%$ of the single user performance, whereas TDMA offers better robustness with non-zero effective rates at lower SNRs. Future works on this topic should study the impact of channel estimation errors and time-variability so as to provide a deeper analysis of achievable and effective rates. 


\section{REFERENCES}

[1] M. Stojanovic and P.-P. J. Beaujean, "Acoustic Communication," in Springer Handbook of Ocean Engineering, M. R. Dhanak and N. I. Xiros, Eds. Springer International Publishing, 2016, pp. 359-386.

[2] T. Melodia, H. Kulhandjian, L.-C. Kuo, and E. Demirors, "Advances in Underwater Acoustic Networking," in Mobile Ad Hoc Networking, S. Basagni, r. Conti, S. Giordano, and I. Stojmenovic, Eds. John Wiley \& Sons, Inc., 2013, pp. 804-852. [Online]. Available: http: //onlinelibrary.wiley.com/doi/10.1002/9781118511305.ch23/summary

[3] R. Otnes, A. Asterjadhi, P. Casari, M. Goetz, T. Husoy, I. Nissen, K. Rimstad, P. van Walree, and M. Zorzi, Underwater Acoustic Networking Techniques. Springer Briefs in Electrical and Computer Eng., 2012.

[4] S. E. Cho, H. C. Song, and W. S. Hodgkiss, "Multiuser acoustic communications with mobile users," The Journal of the Acoustical Society of America, vol. 133, no. 2, pp. 880-890, Feb. 2013. [Online]. Available: http://asa.scitation.org/doi/10.1121/1.4773267

[5] J. Huang, S. Zhou, and Z. Wang, "Performance Results of Two Iterative Receivers for Distributed MIMO ODFM with Large Doppler Deviations," IEEE J. of Oceaninc Eng., vol. 38, no. 2, pp. 347-357, April 2013.

[6] Z. Wang, S. Zhou, J. Catipovic, and P. Willett, "Asynchronous Multiuser Reception for OFDM in Underwater Acoustic Communications," IEEE Transactions on Wireless Communications, vol. 12, no. 3, pp. 10501061, Mar. 2013.

[7] P. A. van Walree, F.-X. Socheleau, R. Otnes, and T. Jenserud, "The watermark benchmark for underwater acoustic modulation schemes," IEEE
Journal of Oceanic Engineering, vol. 42, no. 4, pp. 1007-1018, Oct 2017, software available at https://www.ffi.no/en/research/watermark.

[8] T. M. Cover and J. A. Thomas, Elements of Information Theory. John Wiley \& Sons, 2006

[9] V. Belmega, On Resource Allocation Problems in Distributed MIMO networks. PhD. Thesis, Université Paris Sud - Paris XI, 2010

[10] P. Bouvet, Y. Auffret, D. Munck, A. Pottier, G. Janvresse, Y. Eustache, P. Tessot, and R. Bourdon, "Experimentation of mimo underwater acoustic communication in shallow water channel," in OCEANS 2017 . Aberdeen, June 2017, pp. 1-6.

[11] G. Caire, G. Taricco, and E. Biglieri, "Bit-interleaved coded modulation," IEEE Transactions on Information Theory, vol. 44, no. 3, pp. 927-946, May 1998.

[12] H. Rohling, "Radar cfar thresholding in clutter and multiple target situations," IEEE Transactions on Aerospace and Electronic Systems, vol. AES-19, no. 4, pp. 608-621, July 1983.

[13] C. Laot, A. Glavieux, and J. Labat, "Turbo equalization : adaptive equalization and channel decoding jointly optimized," IEEE Journal on Selected Areas in Communications, vol. 19, no. 9, pp. 1744 - 1752, 2001.

[14] P. van Walree, Channel sounding for acoustic communications: techniques and shallow-water examples. Research Report, Norwegian Defence Research Establishment (FFI), Kjeller, Norway, 2011.

[15] P. van Walree, P. Jenserud, and M. Smedsrud, "A Discrete-Time Channel Simulator Driven by Measured Scattering Functions," IEEE J. Selected Areas in Communications, vol. 26, no. 9, pp. 1628-1637, 2008.

[16] P. Bouvet and Y. Auffret, "On the achievable rate of multipleinputmultiple-output underwater acoustic communications," IEEE Journal of Oceanic Engineering, pp. 1-12, 2019. 